\title{
Effect of ladle outlet geometry on internal porosity in gravity casting automotive brackets: An experimental investigation
}

\author{
Jaehoon Choi ${ }^{1}$, Hoyoung Hwang ${ }^{2,3}$, and *Shinill Kang ${ }^{1,4}$ \\ 1. School of Mechanical Engineering, Yonsei University, 50 Yonsei-ro, Seodaemun-gu, Seoul, 03722, Korea. \\ 2. Digital Manufacturing Process Group, Korea Institute of Industrial Technology, 113-58, Seohaean-ro, Siheung-si, Gyeonggi-do, 15014, Korea \\ 3. Industrial Technology, Korea University of Science and Technology, 217, Gajeong-ro, Yuseong-gu, Daejeon, 34113, Korea \\ 4. National Center for Optically-assisted Mechanical Systems, Yonsei University, Seoul, 03722, Korea
}

\begin{abstract}
This study investigated whether the pouring ladle's outlet shape could reduce the porosity of aluminum alloy casting products and whether it could be applied to mass production of automotive brackets based on computer simulations and experiment. The filling behaviors of the melt were simulated to compare the flow characteristics of the molten metal poured from the conventional ladle and the proposed ladle. Results show that for the conventional ladle, the pouring metal is V-shaped, while it is relatively circular and poured at a more constant rate in the proposed ladle. CT scans reveal that the proposed ladle reduces the porosity of the cast product. The proposed ladle resulted in an average of 30 fewer pores, a $299.56 \mathrm{~mm}^{3}$ smaller average total pore volume, and a $0.041 \%$ lower difference in average porosity. It is concludes that the proposed ladle can be used in the gravity casting process. By changing the ladle outlet shape can reduce the casting failure rate of massproduced automobile bracket
\end{abstract}

Key words: aluminum alloy; pouring ladle; gravity casting; porosity

CLC numbers: TG232.7 Document code: A Article ID: 1672-6421(2020)01-056-05

\begin{abstract}
$\mathrm{A}$ luminum alloys have excellent specific strength and are widely used as lightweight materials in transport and machinery applications. Aluminum parts are mainly manufactured using a casting process. During the casting processes such as high pressure die casting, low pressure die casting, squeeze casting, and vacuum suction casting, an external pressure is applied to the melt flow when molten metal is injected into the mold. These processes can be distinguished by sand casting, and tilt casting, in which the melt is filled only by gravity, without any outside pressure $^{[1-8]}$.

Many studies have investigated whether casting defects in products can be reduced in gravity casting using permanent molds by controlling the flow and solidification of molten metal. However, the ladle used to pour molten metal in the gravity casting process was
\end{abstract}

\footnotetext{
*Shinill Kang

Male, Ph. D, Professor, a Director of the National Center for Optically-assisted Mechanical Systems (ERC), Yonsei University, Korea. He has published more than 100 peer reviewed SCl journal papers and a book, "Micro/Nano Replication: Processes and Applications". His research interests include nano/ micro fabrication, micro optics and nano plasmonics.
}

E-mail: snlkang@yonsei.ac.kr

Received: 2019-10-22; Accepted: 2019-12-18 not generally considered important and only a few researchers conducted simulations ${ }^{[9-14]}$. Conventional ladles are generally cylindrical and are empirically designed taking into account casting process parameters such as pouring location, convenience and pouring volume. However, the size of the pouring ladle and the shape of the outlet can increase the trapping of the bubbles into the molten metal during pouring, which can cause bubble defects and adversely affect the mechanical properties ${ }^{[15-18]}$.

Lekakh et al. ${ }^{[19]}$ studied the flow of molten metal in ladles during continuous casting using simulations. Gongfa et al. ${ }^{[20]}$ studied ladles with an insulation layer. Kabakov et al. ${ }^{[21]}$ examined the importance of process features for a $350 \mathrm{t}$ heavy ladle and examined the effects of ladle shape. Majidi et al. ${ }^{[22]}$ asserted that air insulation has to be avoided during molding, by analyzing porosity in simulations using water, not metal, as the insulating material.

In this study, a permanent mold was used to conduct the casting experiment, because sand casting can result in extra porosity due to the chemical reaction of the coated sand with the molten metal and the mold wash or sand mold ${ }^{[23]}$. The casting process simulation was 
performed using software based on the SOLA-VOF method ${ }^{[24]}$, to examine the effects of the shapes of the proposed and conventional ladles. Figure 1(a) is an image of molten metal being poured into the permanent mold using the conventional ladle. Figure $1(\mathrm{~b})$ is a cross-sectional image of $\mathrm{A}-\mathrm{A}^{\prime}$ showing a wide $\mathrm{V}$-shaped spread of the metal. When pouring the molten metal, it can be expected that the temperature loss will become higher as the melt-air contact surface area increases. Figure 1(c) is an image of molten metal in a circular shape being poured using the proposed ladle.

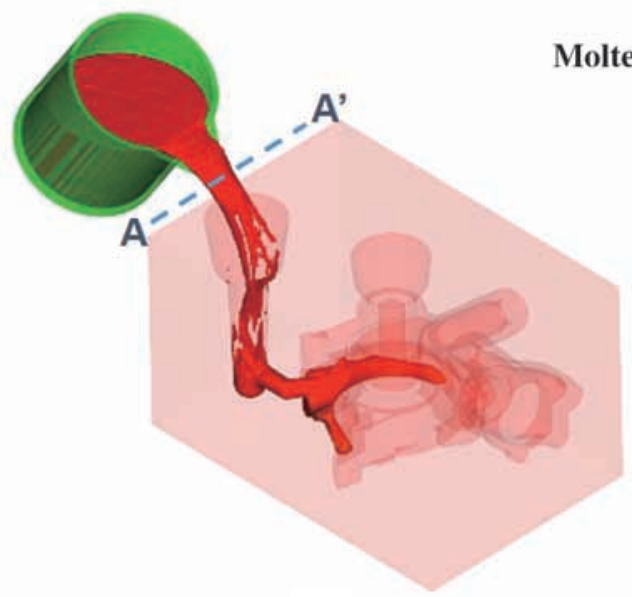

(a)

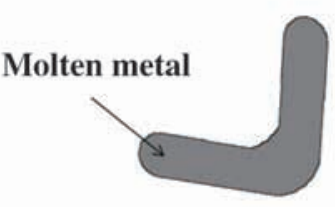

(b)

Top-view of cross-section of $\mathbf{A}-\mathbf{A}^{\prime}$

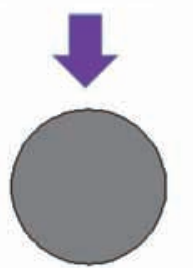

(c)

Smooth circular shape

Fig. 1: (a) Gravity casting schematic; (b) Top view of the metal cross-section A-A' showing the wide V-shaped spread of the molten metal; (c) An image of molten metal in a smooth circular shape being poured with the proposed ladle ${ }^{[25]}$

The gravity casting of the automobile bracket was performed using the proposed ladle and the conventional ladle. Comparative analysis of the two products was carried out based on computed tomography (CT) images, which showed that the proposed ladle reduced the occurrence of porosity defects.

\section{Casting simulation}

A simulation was conducted in which the ladle was $80 \%$ filled with molten AC3A aluminum alloy and fixed at the 10 $\mathrm{cm}$ above the upper part of the mold. The molten metal was poured into the mold while rotating the ladle from $0^{\circ}$ to $100^{\circ}$ for $6.0 \mathrm{~s}$ with the ladle outlet as the rotation reference point. The initial temperature of the molten metal in the ladle was $720{ }^{\circ} \mathrm{C}$. Figure 2 shows the melt images filling the mold. The proposed ladle pours at a relatively uniform rate. In the conventional ladle, a small amount of molten metal was initially poured, and a very large amount was poured from about $3.0 \mathrm{~s}$.

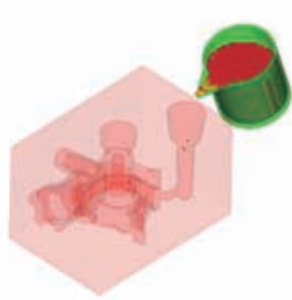

$0.8 \mathrm{~s}$

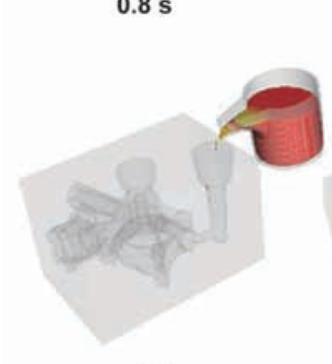

$0.3 \mathrm{~s}$

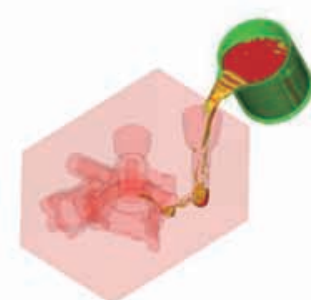

$2.0 \mathrm{~s}$

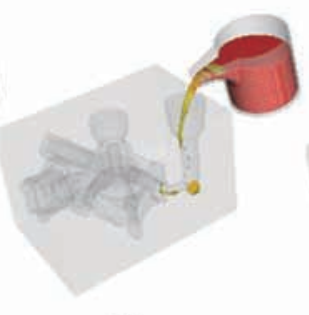

$0.5 \mathrm{~s}$

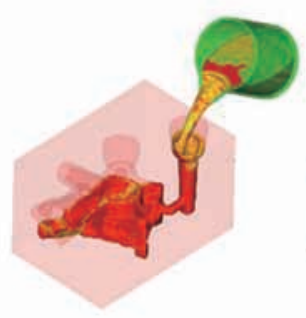

$4.5 \mathrm{~s}$

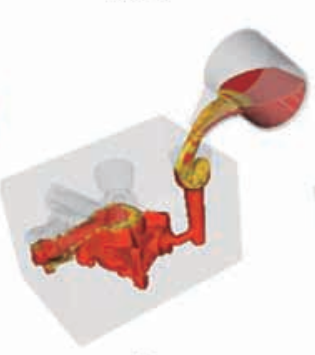

$4.0 \mathrm{~s}$

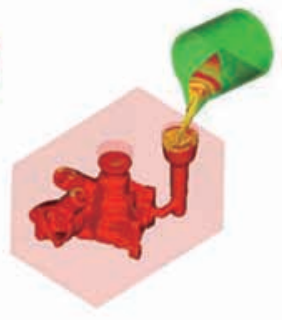

$5.0 \mathrm{~s}$

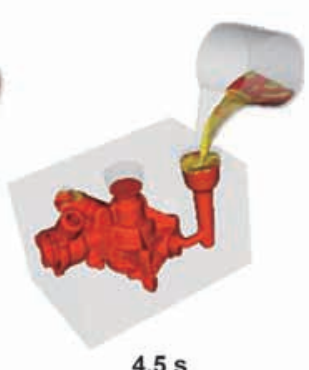

$4.5 \mathrm{~s}$

Fig. 2: Simulated filling behavior of molten aluminum flowing at tilting times from 0.3 to $5.0 \mathrm{~s}$ for the conventional (top) and proposed (bottom) ladle 
Figure 3 shows the average temperature of the melt poured into the mold as a result of the flow simulation. The proposed ladle maintains the temperature of molten metal poured into the mold from the beginning, but the conventional ladle, in which only a small amount of molten metal is poured at the beginning of pouring, cools down quickly. In both ladles, the average temperature of the poured molten metal has a small drop between 1.5 and $3 \mathrm{~s}$ but falls faster after $3 \mathrm{~s}$. As shown in Fig. 3, the temperature drop of the case of the proposed ladle is smaller than that of the conventional ladle. "The smaller the temperature decrease, the better the thermal energy is preserved" is based on the fact that the average temperature of the melt poured into the mold by the proposed ladle is relatively higher than the conventional ladle. This means that the thermal energy of the melt poured from the ladle into the mold is well preserved.

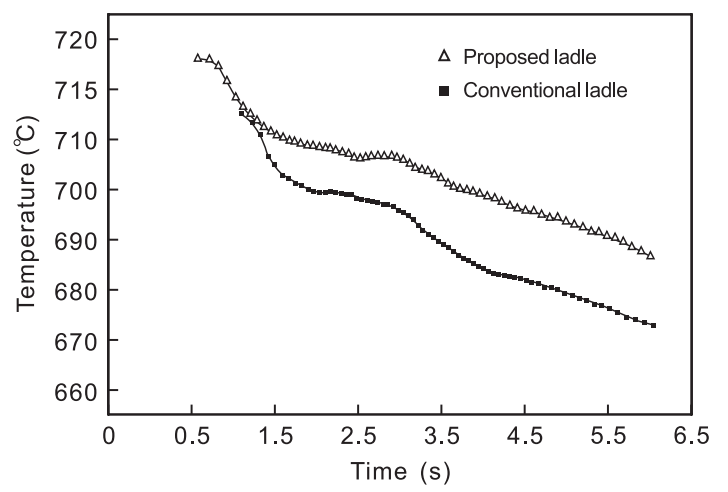

Fig. 3: Average temperature of the melt poured into the mold as a result of flow simulation

When molten metal is poured into the mold, the difference in contacting area with the ambient air arises due to the outlet shape of the ladle. In the conventional ladle, the V-shaped outlet causes a large amount of energy loss due to the large

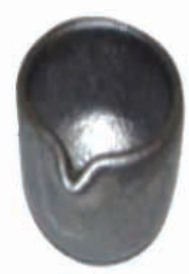

(a)

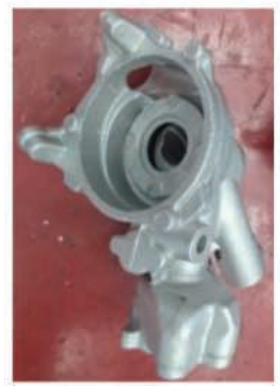

(c)

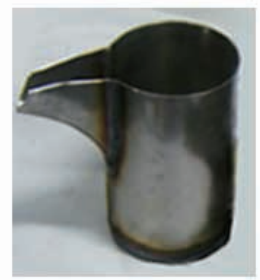

(b)

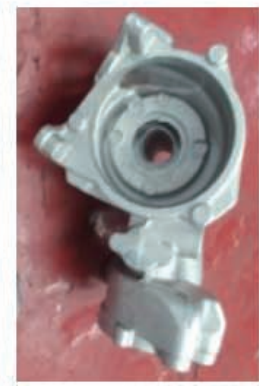

(d) contacting area with the ambient air when the molten metal is poured into the mold. While, in the case of the proposed ladle, since the shape of pouring molten metal is close to the circle, the contacting area with the surrounding air is reduced to minimize the loss of thermal energy during pouring. When using the proposed ladle, we found that the temperature decrease was smaller. It is confirmed that the proposed ladle is much better than the conventional ladle as thermal energy is well preserved for a long time.

As is known, a large amount of air can be accumulated in the molten metal as it is poured from the ladle into the mold. However, in the proposed ladle, less turbulence occurs during the pouring of the molten metal, and bubble defects are expected to be reduced because the temperature of the molten metal poured into the mold is maintained at a higher temperature than that of the conventional ladle.

\section{Experimental methods}

\subsection{Gravity casting process}

A permanent mold was used in the casting experiment with the gravity casting process under the same conditions applied in the simulation, with the two different ladles. The experiment was performed after 10 test castings. The initial temperature of the mold is $200{ }^{\circ} \mathrm{C}$. The aluminum alloy was melted in an electric furnace at $720^{\circ} \mathrm{C}$. Ten experimental specimens were fabricated, five of which were made using the proposed ladle, and the other five were made using the conventional ladle. Figures 4(a) and 4(b) show the conventional and proposed ladle designs, respectively. Figure 4(c) shows a sample specimen made using a conventional ladle, and (d) shows a sample specimen made using the proposed ladle. Figure 4(e) shows an image with the permanent mold open.

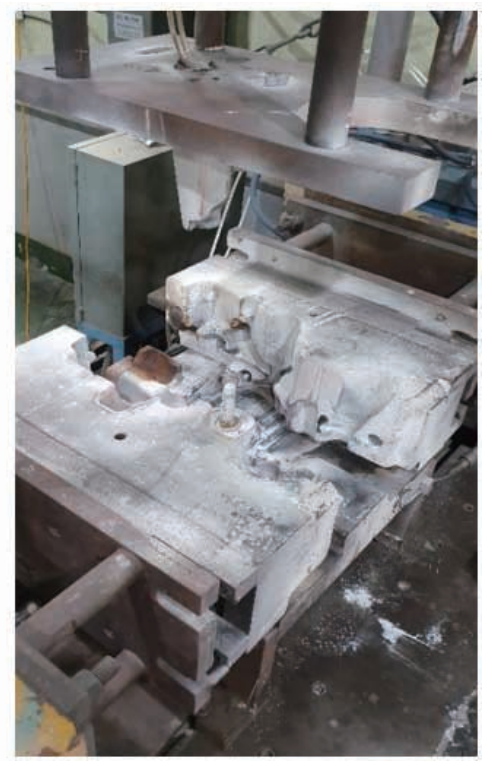

(e)

Fig. 4: Experimental casting using a gravity casting process: (a) a conventional ladle; (b) the proposed ladle; (c) a sample specimen made using the conventional ladle; (d) a sample specimen made using the proposed ladle; (e) an image of the open permanent mold 


\subsection{Computed tomography (CT) analysis}

Industrial $\mathrm{CT}$ is a noninvasive computer imaging technique to analyze several cross-sectional X-rays in order to reveal minute details within a specimen as described in the study of Wits et al. ${ }^{[26]}$. A set of two-dimensional projection images are obtained during a full rotation of a specimen and subsequently threedimensional images are reconstructed from two-dimensional serial computerized tomographic scan data. The reconstructed three-dimensional images of the specimen consist of volumetric pixels (voxels). In this study, the CT scans were performed with RayScan 250 (Rayscan, Germany). The voltage and current of X-ray sources were $180 \mathrm{kV}$ and $290 \mu \mathrm{A}$, respectively. The voxel sizes were $276.41 \mu \mathrm{m}$.
Figure 5 shows the reconstructed three-dimensional (3D) CT images of casting specimens by the conventional and proposed ladles, and the two-dimensional (2D) CT slice images. The color bar indicates the volume of each pore in cubic micrometers. The black part inside the product in the 2D CT image shows the pores inside the specimen. On average, specimens produced using the conventional and proposed ladles have 67 and 37 pores, respectively, as shown in Fig. 6(a), and the total volume of the pores was 673.12 and 373.56 $\mathrm{mm}^{3}$, respectively, as shown in Fig. 6(b). The pore volume fraction (pore volume/total product volume) produced by the conventional and proposed ladle was $0.093 \%$ and $0.052 \%$, respectively, as shown in Fig. 6(c).
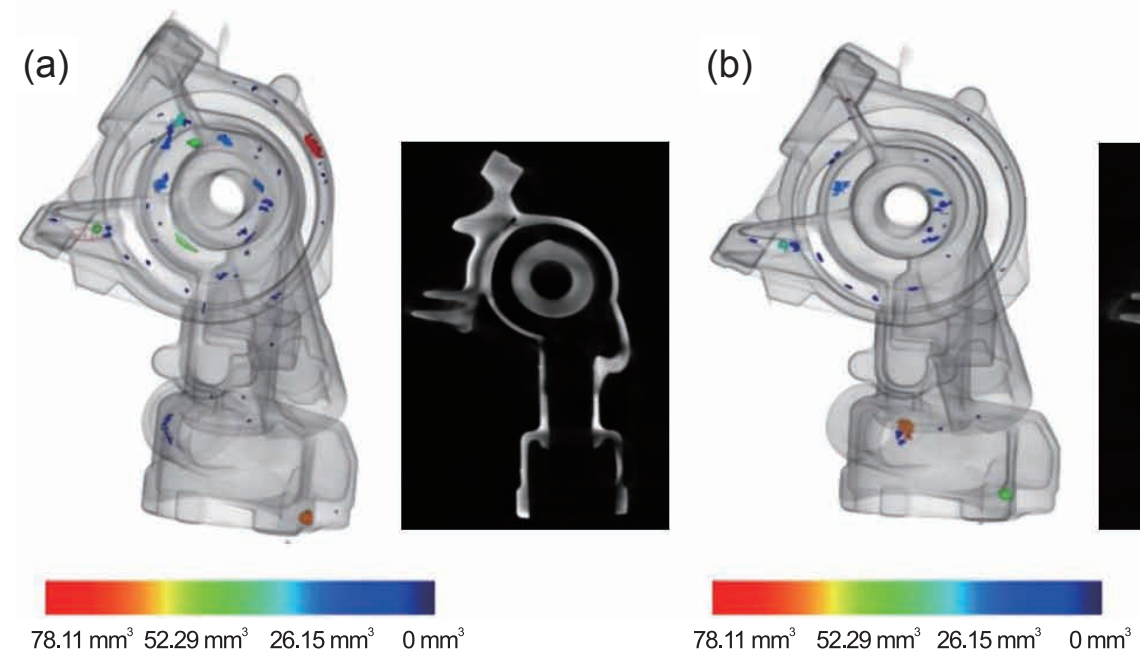

$78.11 \mathrm{~mm}^{3} 52.29 \mathrm{~mm}^{3} \quad 26.15 \mathrm{~mm}^{3} \quad 0 \mathrm{~mm}^{3}$

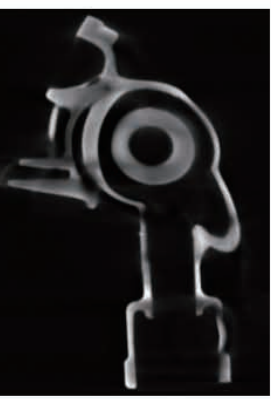

Fig. 5: Reconstructed 3D CT images of cast specimens and the 2D CT slice images by conventional (a) and proposed (b) ladle
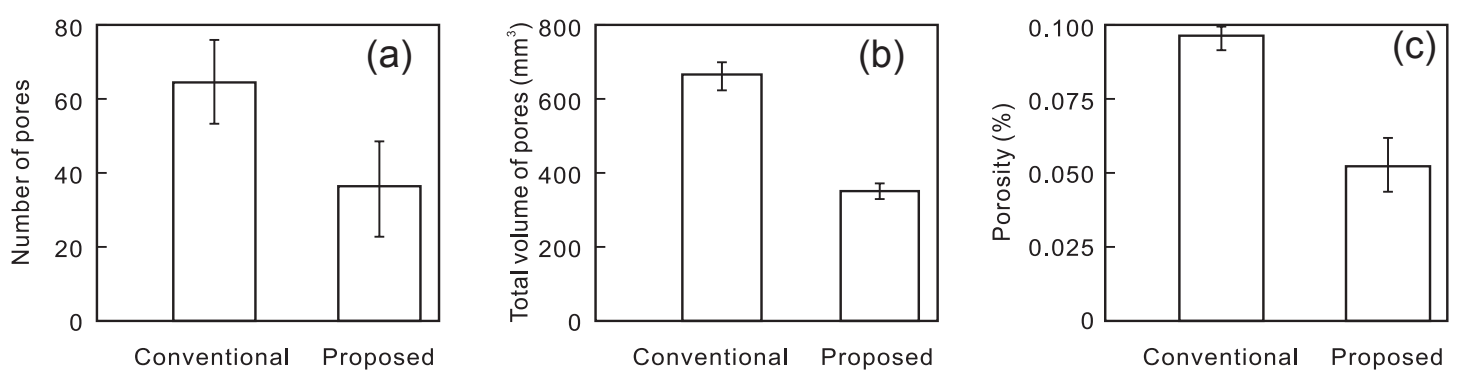

Fig. 6: Analysis of the number of internal pores (a), total volume of internal pores (b), and the internal porosity (c) based on CT scanning images

\section{Conclusion}

This study examined the effect of ladle outlet shape on porosity defects during the gravity casting process to produce an automobile bracket. In computer simulations, the filling behavior of molten aluminum alloys is such that for the conventional ladle, the pouring metal is V-shaped, while for the proposed ladle, the pouring metal is relatively circular and poured at a more constant rate. Thermal analysis simulations show that the conventional ladle produces a relatively large temperature drop, and the proposed ladle maintains temperature, which reduces the heat loss.

Automobile bracket products were cast using the conventional ladle and the ladle proposed in this study. Compared with the conventional ladle, the proposed ladle resulted in 30 fewer average pores, a $299.56 \mathrm{~mm}^{3}$ smaller average total pore volume, and a $0.041 \%$ less difference in average porosity.

In summary, changing the ladle outlet shape can reduce 
the casting failure rate of mass-produced automobile bracket. Research is currently underway to optimize the shape of the ladle outlet according to the size of the product.

\section{Acknowledgements}

This work was supported by the National Research Foundation of Korea (NRF) (2015R1A5A1037668) and the Ministry of Trade, Industry and Energy (Grant N0002310).

\section{References}

[1] Fan K L, et al. Elevated temperature low cycle fatigue of a gravity casting Al-Si-Cu alloy used for engine cylinder heads. Materials Science \& Engineering A, 2015, 632: 127-136.

[2] $\mathrm{Hu} X \mathrm{G}$, et al. Blistering in semi-solid die casting of aluminum alloys and its avoidance. Acta Materialia, 2017, 124: 446-455.

[3] Qin Xin-yu, Su Yong, Chen Jian, et al. Finite element analysis for die casting parameters in high-pressure die casting process. China Foundry, 2019, 16 (4): 272-276.

[4] Zhao H D, et al. Experimental and numerical analysis of gas entrapment defects in plate ADC12 die castings. Journal of Materials Processing Technology, 2009, 209: 4537-4542.

[5] Makoto Hino, et al. Effect of Surface Treatment on Glossiness of Al-Mg-Zn Alloy. Casting Materials Transactions, 2013, 54 (12): 2238-2244

[6] Yize Li, Haibo Yang, Zhiwei Xing. Numerical simulation and process optimization of squeeze casting process of an automobile control arm. The International Journal of Advanced Manufacturing Technology, 2017, 88: 941-947.

[7] Jun Zheng, Binbin Huang, Xingjian Zhou. A low carbon process design method of sand casting based on process design parameters. Journal of Cleaner Production, 2018, 197: 14081422.

[8] Zbigniew Szklarz, Halina Krawiec, Łukasz Rogal. The effect of vacuum suction casting on the microstructure and corrosion behavior of aluminium alloy. Materials Science \& Engineering B, 2019, 240: 23-32.

[9] Campbell J, et al. Influence of Casting Technique and Hot Isostatic Pressing on the Fatigue of an Al-7Si-Mg Alloy. Metallurgical and Materials Transactions A, 2001, 32: 349.

[10] Ananda Rao G, et al. Solidification phenomena and reduction of skull in the ladle using finite difference method and Matlab. Journal on Mechanical Engineering, 2012, 2 (4): 31-39.

[11] Zabolotsky A. V. Modeling of the temperature field of the casting ladle lining. Journal of Engineering Physics and Thermophysics, 2011, 84 (2): 342-347.

[12] Jančar $D$, et al. Utilization of casting ladle lining enthalpy for heating gas savings in the course of ladle preheating. Metalurgija, 2014, 53 (2): 159-162.
[13] Kai Jin, et al. Modeling and measurements of multiphase flow and bubble entrapment in steel continuous casting. Metallurgical And Materials Transactions B, 2016, 47 (1): 548565.

[14] Homayonifar P, et al. Numerical modeling of splashing and air entrapment in high-pressure die casting. The International Journal of Advanced Manufacturing Technology, 2008, 39: 219-228.

[15] Vijayaram T R, et al. Numerical simulation of casting solidification in permanent metallic molds. Journal of Materials Processing Technology, 2006, 178: 29-33.

[16] Papworth A, et al. The disruption of oxide defects within aluminum alloy castings by the addition of bismuth. Materials Letters, 1998, 35: 202-206.

[17] Murat Tiryakioğlu. Pore size distributions in AM50 Mg alloy die castings. Materials Science and Engineering A, 2007, 465: 287-289.

[18] Mousavi Anijdana S H, et al. The effect of pouring temperature and surface angle of vortex casting on microstructural changes and mechanical properties of $7050 \mathrm{Al}-3 \mathrm{wt} \% \mathrm{SiC}$ composite. Materials Science \& Engineering A, 2018, 737: 230-235.

[19] Lekakh $S \mathrm{~N}$, et al. Effect of ladle, tundish and mold design on melt flow parameters. In: Proc. Association for Iron \& Steel Technology, 2014: 1881-1891.

[20] Gongfa Li, et al. Numerical simulation of temperature field and thermal stress field in the new type of ladle with the nanometer adiabatic material. Advances in Mechanical Engineering, 2015, 7 (4): 1-13.

[21] Kabakov Z K, et al. Reducing the loss of heat from steel in steel-pouring ladles. Metallurgist, 2013, 56: 9-10.

[22] Seyyed H M, et al. Modelling of air entrainment during pouring of metal castings. International Journal of Cast Metals Research, 2017, 30 (5): 301-315.

[23] Melendez A J, et al. Modelling of reoxidation inclusion formation in steel sand casting. International Journal of Cast Metals Research, 2013, 23(5): 278-288.

[24] Hong $\mathrm{J} \mathrm{H}$, et al. Optimum design of casting mould by gas porosity prediction module in numerical simulations. In: Proc. Modeling of Casting, Welding and Advanced Solidification Processes - XI, Opio, France, 28 May, 2006: 611.

[25] Jaehoon Choi, et al. A design methodology for casting ladle to minimize air entrapment and oxide inclusion: An application to permanent mold casting process. Materials Transactions, 2019, 60 (12): 2562-2568.

[26] Wits W W, Carmignato S, Zanini F, et al. Porosity testing methods for the quality assessment of selective laser melted parts. CIRP Annals - Manufacturing Technology, 2016, 65: 201-204. 